A. Brancelj, L. De Meester \& P. Spaak (eds), Cladocera: The Biology of Model Organisms.

(c)1997 Kluwer Academic Publishers. Printed in Belgium.

\title{
Offspring size in Daphnia: does it pay to be overweight?
}

\author{
Maarten Boersma \\ Max-Planck-Institut für Limnologie, Postfach 165, D-24302 Plön, Germany \\ (e-mail: boersma@mpil-ploen.mpg.de)
}

Key words: Life history, food

\begin{abstract}
Variation in offspring size and number has been described for a wide range of organisms. In this study I investigated the relationship between resource level of the mother and size of her offspring in the cladoceran Daphnia magna, in order to assess whether offspring produced at different food levels are optimal in size for these food levels. Optimal offspring size was defined as the size of offspring that yields the highest parental fitness (i.e. offspring of optimal size have the highest juvenile fitness per unit maternal effort invested in them). I observed that especially at the higher food levels, daphnids produced offspring that are larger than the computed optimal offspring size at these food levels. I interpret this as a mechanism to avoid starvation of neonates in the case of suddenly deteriorating food conditions.
\end{abstract}

\section{Introduction}

One of the general axioms in the theories of life-history evolution is that selection should favour those parental strategies which maximize parental fitness (e.g. in the determination of the number and size of offspring produced, Lack (1947)). According to the classic paper of Smith \& Fretwell (1974), maximizing parental fitness will lead to a single optimal investment per offspring for any given environment, provided that (1) a trade-off exists between size and number of individual offspring, i.e. the amount of energy invested in reproduction per breeding attempt is fixed; and (2) that as energy expended on individual offspring increases, fitness of individual offspring increases. The optimal investment per offspring is characterised by the highest fitness of individual juveniles per unit effort put into these animals. Changes in either total resource acquisition, or in the proportion of resources which is allocated to reproduction, should not change this optimal investment per offspring, as the total effort per breeding attempt does not influence the parental fitness-function (Smith \& Fretwell, 1974). In planktonic cladocerans such as Daphnia, the trade-off between size and number of offspring has been repeatedly observed (e.g.
Ebert, 1993; Boersma, 1997), but is often masked by differences in total available resources for reproduction (see van Noordwijk \& de Jong, 1986). Moreover, as daphnids live in changing environments with respect to food conditions, the resource acquisition of the adults may be used as an estimate of environmental circumstances which their offspring will encounter after birth. Adults and juveniles generally show a large overlap in their resources, and the mothers adjust the size of their offspring according to their own resource availability, resulting in a relationship between resource level and egg size in cladocerans, characterized by larger offspring produced at lower food levels (e.g. Tessier \& Consolatti, 1991; Glazier, 1992; Guisande \& Gliwicz, 1992; Ebert, 1994). This relationship is easy to understand intuitively, as offspring produced by females grown at low food levels should have more reserves to withstand the concurrent food conditions than when food is in ample supply and juveniles can start feeding immediately. Thus far, however, to my knowledge only one study exists which tries to link the investment in individual offspring with their successive fitness in a more formal way, in order to study whether the offspring produced at different food levels are optimal for the ambient environmental conditions (Tessier \& Con- 
solatti, 1989).These authors, however, only described a theoretical framework, and made no attempt to quantify optimal offspring sizes. In the present study, I set out to perform this quantification. I collected neonates with different initial weights produced by first adult instar females (Boersma, 1997), and cultured them at four different food levels to assess the optimal offspring weight under these conditions. These values of optimal offspring weight were then compared to observed offspring weights.

\section{Materials and methods}

\section{Offspring fitness}

Fitness, usually defined as the average number of offspring produced by individuals with a certain genotype, relative to the number produced by individuals with other genotypes (e.g. Ridley, 1993), is in the current literature often represented by the intrinsic rate of population increase, $r$ (e.g. Stearns, 1992). Most laboratory studies on cladocerans use the Euler-Lotka equation to estimate $r$ iteratively (e.g. Ebert \& Jacobs, 1991; Spitze, 1992; Weider, 1993; De Meester, 1994; Spaak \& Hoekstra, 1995; Lampert \& Trubetskova, 1996). Often, these values of the intrinsic rate of population increase are computed using individual animals (Weider, 1993; De Meester, 1994; Spaak \& Hoekstra, 1995). This causes problems, as doing so makes it impossible to find negative values of $r$, except for the infinite negative, when an animal does not reproduce at all. The production of one single egg in the animal's life-time will lead to an $r$-value equal or larger than zero. Moreover, even if $r$ equals zero, this does not mean that fitness equals zero, as the individual is still capable of replacing itself, leading to a stable population density. It could therefore be argued that the intrinsic rate of population increase is not suitable as a fitness measure, but that rather $\mathrm{e}^{r}(\lambda)$ would be the appropriate fitness measure, as this value equals zero when $r$ approaches negative infinity. However, this fitness measure essentially only rescales the observations, and hence does not solve the above mentioned problems with $r$, as now values between 0 and 1 will be absent. This interval of $0<\mathrm{e}^{r}<1$ is of lesser interest when assessing optimality of offspring size, because when population growth rates are less then 1 , the population will die out. Therefore, the domain of the optimization problem for realistic purposes has a lower limit of $\mathrm{e}^{r}=0$, which will result in a stable pop- ulation. Moreover, given the nature of $r$, with values usually small and close to zero, the distinction between $r$ and $\mathrm{e}^{r}$ is rather subtle, as $\mathrm{e}^{r}$ shows a linear relationship with $r$ for small intervals. To allow comparison with other studies, and given the considerations above $r$, was used as a measure of fitness in this study.

Laboratory derived $r$-values as a measure of fitness do not incorporate mortality occurring in the field. Evolution of egg size obviously has occured with mortality sources, such as predation, present. Size differences between offspring are, however, usually not large, and thus far it has been very difficult to properly estimate differences in mortality in the field between animals with such subtle size differences (e.g. Boersma et al., 1996). As these differences is size-specific mortality are unknown, and are likely to change within a growing season, it is difficult to incorporate field-mortality in the computation of optimal offspring size. One way to circumvent this is to estimate fitness under different assumptions of juvenile size-selective mortality. However, as very little is known about this size-selective juvenile mortality, the power of this analysis would be limited. Alternatively, optimal offspring sizes can be computed under the assumption that mortality differences are non-existent. Then, from the computed optimal offspring sizes and the measured actual offspring sizes, the size-specific mortality needed to make the observed offspring size the optimal offspring size can be computed. The validity of this set of size-specific mortality assumptions could then be assessed by field observations and experiments.

A significant correlation has been found between juvenile growth rate (somatic growth between birth and maturity) and the intrinsic rate of population increase, $r$ (Lampert \& Trubetskova, 1996). As juvenile growth rates are easier and quicker to establish, I collected one individual per experimental vessel when the animals were five days old, and again upon reaching maturity. These animals were used to establish dry-weights in order to estimate growth rates. In order to estimate the exact relation between juvenile growth and $r$ under the conditions of the present study, I also established $r$-values for animals placed in 20 experimental chambers per series; these animals were cultured until they released their second broods. Development times and number of juveniles produced were used to estimate $r$ by solving the Euler-Lotka equation iteratively. The relationship between growth rates and $r$-values computed for these individuals was then used to establish $r$-values for all experimental animals. 


\section{Offspring weight offspring fitness}

The Daphnia magna clone used in this study has been kept in the laboratory for many years, and was originally collected from a pond in Frankfurt, Germany. From a stock culture, juvenile animals were collected randomly. These animals were placed individually in $120 \mathrm{ml}$ flow-through chambers, with a flow rate of 1 litre $\mathrm{d}^{-1}$, and fed a Scenedesmus acutus suspension with an algal carbon content of $0.8 \mathrm{mg} \mathrm{C}^{-1}$ at $20{ }^{\circ} \mathrm{C}$, under continuous light conditions. The algae were grown in 3-litre chemostats in Chu-12 medium (Lampert et al., 1988). Food suspensions were prepared daily by adding Scenedesmus to $0.45 \mu \mathrm{m}$ filtered lake water. Algal concentrations were measured spectrophotometrically. The only way to obtain a large set of offspring with different initial weights produced by first adult instar females is to harvest these neonates from animals cultured at different food levels. Therefore, first brood neonates of the animals cultured at $0.8 \mathrm{mg} \mathrm{C}^{-1}$ were collected and these neonates were placed in flow-through chambers at four different food levels: $0.1 ; 0.2 ; 0.4$ and $1.0 \mathrm{mg} \mathrm{C}^{-1}$. Each chamber contained 5-10 individuals. Upon reaching maturity, these animals were measured, the length of the neonates produced by these animals was established, and two or three neonates from every chamber were weighed to establish dry weight of individual offspring. If possible, five other neonates from each chamber were collected and placed in flow-through chambers to assess their fitness. Neonates produced at the different food levels were divided evenly across all four food levels mentioned above.

The assumption behind the Smith \& Fretwell (1974) model is that offspring fitness increases monotonically with offspring weight up to an asymptote (i.e. increasing per-offspring investment results in diminishing fitness gains). Hence, I iteratively fitted the three parameter model proposed by Tessier \& Consolatti (1991) to describe the dependence of offspring fitness $(f)$ on offspring investment $(n)$ :

$$
f=f_{\mathrm{m}}\left[1-\mathrm{e}^{-k\left(n-n_{\mathrm{o}}\right)}\right],
$$

where $n_{\mathrm{o}}=$ the minimum viable neonate mass, $f_{\mathrm{m}}=$ the maximum fitness level, and $k$ the rate of rise of fitness with neonate mass (see also Winkler \& Wallin, 1987). As pointed out by Tessier $\&$ Consolatti, $f_{\mathrm{m}}$ will be most sensitive to changes in food concentration in the environment, with lower food levels leading to lower values of $f_{\mathrm{m}}$. However, the optimal solution in the offspring weight-offspring number trade-off is independent of the value of $f_{\mathrm{m}}$ (Parker \& Begon, 1986), and therefore $f_{\mathrm{m}}$ was estimated separately, being the average fitness of all animals with a birth-weight larger than $10 \mu \mathrm{g}$.

\section{Egg quality}

The premise for the approach taken in this paper to be valid is that, apart from the differences in size between the offspring, no quality differences between offspring produced at different food levels should exist. This was tested for the four different food levels, in an experimental set-up identical to the one described above. Experimental animals were collected, and placed in flow-through chambers at the four food levels. Each chamber contained 10 individuals, with 22 replicate chambers per food level. Once these animals reached maturity they were harvested, the eggs produced by these animals were separated from the mothers, and analysed for volume, dry weight, carbon content and fat content. Observations were made every 12 hours, so the average age of these eggs was six hours. The analysis of carbon content and fat content are mutually exclusive so two different sets of samples had to be prepared, one to be analysed further for ash free dry weight and carbon content of the eggs, and the other one to be used in the analysis of total fat content of the eggs. A minimum of five eggs per sample were collected, if possible from the same mother. For the individuals cultured at the lower food levels, however, this was not always possible. Daphnia eggs are nearly spherical in early development, and hence one measured diameter per egg sufficed for the estimation of egg volume (Lampert, 1993). Egg diameter of three eggs per brood was established to the nearest $0.01 \mathrm{~mm}$. For the analysis of dry weight and carbon content, eggs were collected in pre-combusted small silver weighing boats, and dried for 24 hours at $60{ }^{\circ} \mathrm{C}$. These samples were stored in a desiccator, weighed to the nearest $0.1 \mu \mathrm{g}$ using an electronic microbalance, and subsequently analysed for carbon content.

For the analysis of fat (triglyceride) content, eggs were collected and stored at $-20{ }^{\circ} \mathrm{C}$ in $100 \mu \mathrm{l}$ phosphate buffered saline solution (Sigma; P-4417). They were homogenized, and the fat content was established using triglyceride (GPO-Trinder) reagent (Sigma; Catalogue number 337), which is normally used for the quantitative enzymatic determination of triglycerides in serum or plasma (see also Stibor, 1995). 
Table 1. Quantity differences between eggs produced at different food levels. Four different food levels were tested $(0.1$, $0.2,0.4$, and $\left.1.0 \mathrm{mg} \mathrm{C}^{-1}\right)$, a total of 70 clutches were analysed. $P$-values of analyses of variance are shown

\begin{tabular}{lc}
\hline Trait & $P$-value \\
\hline Volume $\left(\mathrm{mm}^{3}\right)$ & $<0.001$ \\
Carbon content $(\mu \mathrm{g})$ & $<0.001$ \\
Ash-free dry weight $(\mu \mathrm{g})$ & $<0.001$ \\
Fat $(\mu \mathrm{g})$ & 0.20 \\
\hline
\end{tabular}

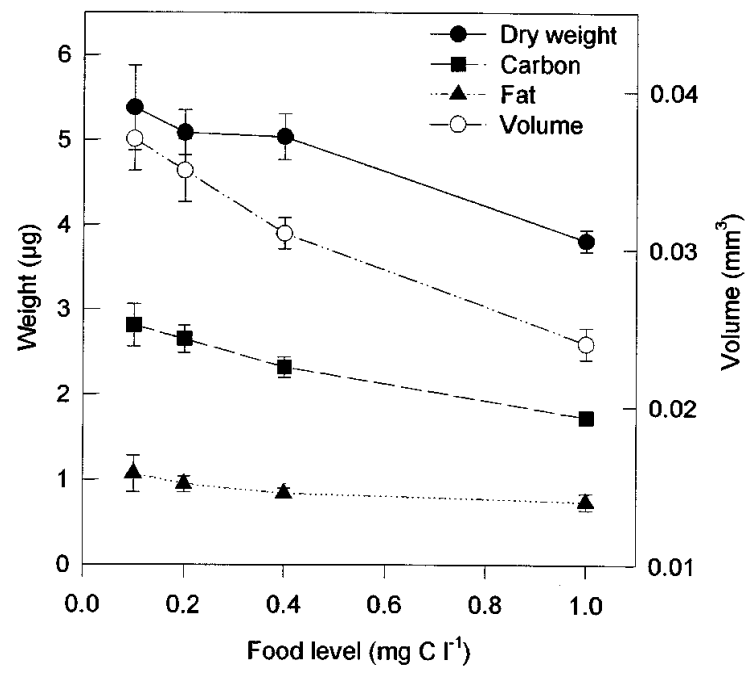

Figure 1. Quality differences between eggs produced at different food levels. Four different food levels were tested $(0.1,0.2,0.4$, and $1.0 \mathrm{mg} \mathrm{C}^{-1}$ ), a total of 70 clutches were analysed. Mean values and standard errors of carbon content $(\mu \mathrm{g})$, ash-free dry weight $(\mu \mathrm{g})$, fat $(\mu \mathrm{g})$ and volume $\left(\mathrm{mm}^{3}\right)$ are shown.

\section{Results}

\section{Egg quality}

Differences in volume, carbon content and ash-free dry weight of eggs produced at the four experimental food levels were highly significant (Table 1), whereas I observed no signifcant differences in fat content of the eggs. The pattern was similar for all four measures: highest values were found for the eggs produced by females grown at the lowest food level, and lowest values were observed for eggs produced by females cultured at $1.0 \mathrm{mg} \mathrm{Cl}^{-1}$ (Figure 1). A two-way ANOVA with food concentration and trait as the grouping variables, and the log-transformed measurements as
Table 2. Summary table of the iterative curve fitting of the relationship between offspring fitness and initial offspring weight (standard error of estimate). The following function was fitted: $f=f_{\mathrm{m}}\left\{1-\mathrm{e}^{-k\left(n-n_{\mathrm{o}}\right)}\right\}$. Total explained variance and significance of the whole model are shown. Values for $f_{\mathrm{m}}$ were determined by averaging the realised fitness of animals with a initial dry weight of more than $10 \mu \mathrm{g}$

\begin{tabular}{lllllll}
\hline Food level & $f_{\mathrm{m}}$ & $k$ & $n_{\mathrm{o}}$ & $r^{2}$ & $N$ & $P$ \\
\hline$\left(\mathrm{mg} \mathrm{C}^{-1}\right)$ & $\left(\mathrm{d}^{-1}\right)$ & $\left(\mu \mathrm{g}^{-1}\right)$ & $(\mu \mathrm{g})$ & & & \\
\hline 0.1 & \multicolumn{6}{c}{ no feasible solution } \\
0.2 & 0.19 & 0.48 & 3.46 & 0.24 & 62 & $<0.001$ \\
& & $(0.25)$ & $(1.49)$ & & & \\
0.4 & 0.26 & 0.54 & 2.79 & 0.22 & 72 & $<0.001$ \\
& & $(0.42)$ & $(2.10)$ & & & \\
1.0 & 0.45 & 0.36 & 1.92 & 0.31 & 82 & $<0.001$ \\
& & $(0.11)$ & $(1.27)$ & & & \\
& & & &
\end{tabular}

the dependent ones showed a non-significant interaction $\left(F_{9,280}=0.205 ; P=0.99\right)$. This means that the relative differences between food levels are similar for the different traits, and implies that no quality differences exist between the food levels.

\section{Offspring weight offspring fitness}

Figure 2 shows the relation between initial offspring weight and the computed values for $r$ from the daily growth increments. Unexpectedly, most data points seem to fall within the saturated part of the curves relating offspring fitness with offspring investment, making it difficult to fit curves of the shape described by Smith \& Fretwell (1974). Especially for animals cultured at the lowest food level, no solution could be found. As a result of the distribution of the data, even the curves fitted through the data of the animals cultured at the higher food levels explain only a small (but significant) portion of the variation (Table 2; Figure 2). With the help of these curves, using the marginal value solution proposed by Smith \& Fretwell (1974) it was possible to estimate the optimal offspring weight at the different food levels (Figure 2). For the food levels 0.2, 0.4 , and $1.0 \mathrm{mg} \mathrm{C}^{-1}$ the optimal offspring weights were computed to be $6.4,5.3$, and $4.6 \mu \mathrm{g}$ dry weight, respectively. The method of estimating optimal birth weight from the fitness profile is in essence an inverse regression problem, and the techniques to assign confidence intervals for the linear case are well-developed. (Sokal \& Rohlf, 1981). In the non-linear case, however, these techniques are less readily available. Since $k$ 


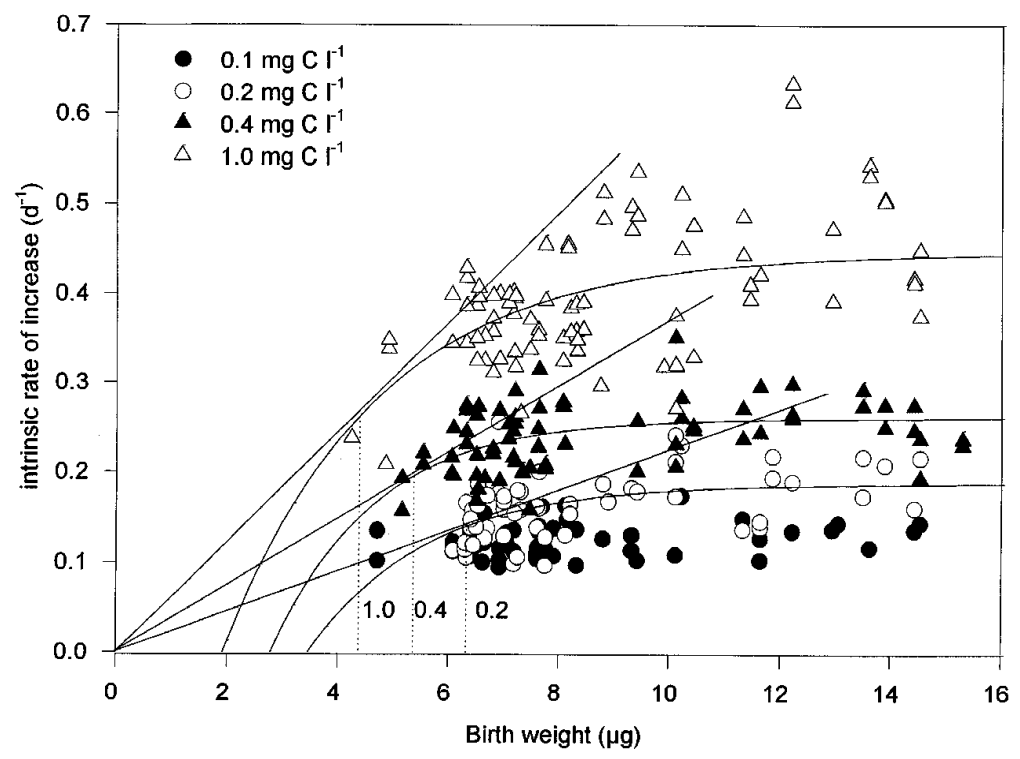

Figure 2. Initial offspring weight $(\mu \mathrm{g})$ and offspring fitness $\left(r, \mathrm{~d}^{-1}\right)$ as computed from the daily growth increments. For the highest three food levels the following functions were fitted: $f=f_{\mathrm{m}}\left\{1-\mathrm{e}^{-k\left(n-n_{\mathrm{o}}\right)}\right\}$ (see also Table 2) in order to estimate the optimal offspring weight at these food levels (indicated on the abscissa).

essentially is the regression coefficient in the current study, I assessed the variability of the estimates of optimal offspring weight by computing the optimal weight at different food levels, with $k \pm$ one standard error. These 'confidence-intervals', obviously asymmetrical around the estimated values, were found to be 5.8-7.7 $(\operatorname{avg}=6.4), 4.4-7.1$ (5.3) and 4.4-5.3 (4.6) for the different food levels. The comparison of the computed values for optimal offspring weights at the different food levels with the weights of the offspring actually produced by these animals revealed that the actual offspring weight, even for the first adult instar mothers used in this study, was larger than the computed optimal offspring weights for these food levels, especially for the animals at the higher food levels (Figure 3). At the lower end of the food concentration spectrum, the differences between the computed optimal and actual offspring weights were smaller.

\section{Mortality}

Fitness, $r$, as established from abbreviated life-tables inadequately describes fitness under field conditions. Mortality in the field is obviously much higher than in the laboratory, as mortality by predation is completely ruled out in the laboratory. This implies that the measurements of $r$ in the laboratory are only related to dif-

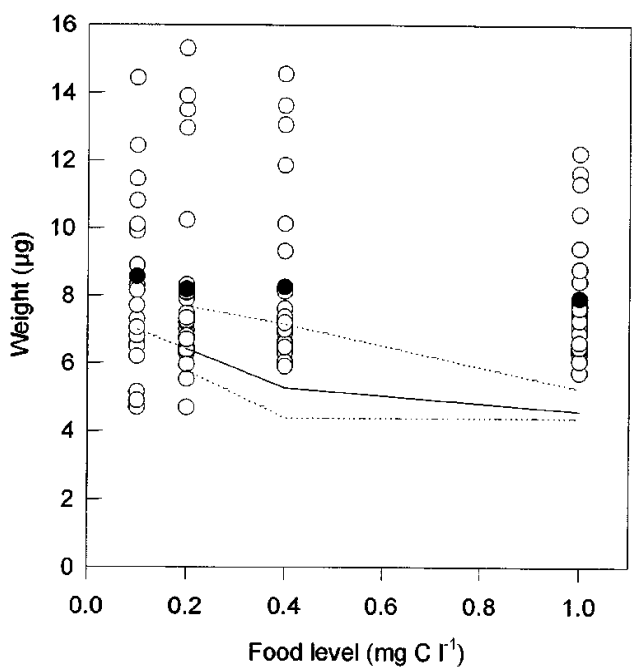

Figure 3. Optimal offspring weight ( $\mu \mathrm{g}$ ) (line) and actual offspring weight $(\mu \mathrm{g})$ (symbols) at different food concentrations (dotted line indicates 'confidence interval' for optimal offspring weight, filled symbols represent average offspring weight at different concentrations).

ferences in food concentration. Size-selective mortality in the field would change the relative $r$ values of the different size classes, which could change the conclusions of the present study considerably. I assessed the pos- 
sible influence of size-selective mortality as follows: first I assumed that the observed average offspring weight produced at the different food levels is actually the optimal offspring weight under these food conditions under natural circumstances. This implies that the curve relating juvenile fitness to offspring weight should be redrawn in such a way that the actual average weight becomes the point on the abscissa at which the line passing through the origin is tangent to the curve (Figure 4). As the value of the maximal attainable fitness, $f_{\mathrm{m}}$, does not influence this point, $f_{\mathrm{m}}$ values were kept constant. The values for $n_{0}$ are most likely only influenced by concurrent food conditions, and not by predatory effects. Hence, the curves were redrawn, with only $k$ fitted, and $f_{\mathrm{m}}$ and $n_{0}$ kept constant. Under the assumption that daily mortality is constant, mortality $(m)$ can be entered in the Euler equation by substituting $l_{x}$, the probability of survival to time $x$, by $\mathrm{e}^{x m}$ (Stearns, 1992). This implies that $m$ and $r$ scale linearly, and hence that the mortality needed to decrease the observed $r$-values in such a way that the observed average weights produced at the different food levels are optimal for these food levels can be computed. Hence, the adjusted $r$-values fall on the line described by the old $f_{\mathrm{m}}$ and $n_{0}$, and the recomputed $k$ (Figure 5). It should be kept in mind that, since $f_{\mathrm{m}}$ was not changed, the absolute value of the mortalities is not of interest. It is the change in mortality rate with changes in initial body mass that is relevant. The calculated decrease in mortality with the increase in birth weight equals $0.006 \mathrm{~d}^{-1} \mu \mathrm{g}^{-1}(r=-0.19 ; P<0.01 ; N=211)$, which implies that individuals with a birth-weight of $6 \mu \mathrm{g}$ should have a mortality in the field, which is $0.054 \mathrm{~d}^{-1}$ higher when compared with animals which are $15 \mu \mathrm{g}$ at birth.

\section{Discussion}

In this study I set out to investigate the relationship between initial weight of neonates of Daphnia magna and their fitness. The main conclusion of this paper should be that this is not an easy task. First of all, as was already indicated in the Materials and Methods section, a measure of juvenile fitness, given the individual culturing set-up as the method of choice in life-history experiments, is difficult to find. Although the vast majority of papers do take the intrinsic rate of population increase, $r$, as a fitness measurement it should be reiterated that fitness equals zero when $r$ equals negative infinity, i.e when the animal dies before reproducing. Values between negative infinity and 0 (when an animal produces exactly one offspring during its life-time) are not possible in such experimental systems. $\mathrm{e}^{r}(\lambda)$ would be a better fitness measurement, as this value equals zero when the animal does not reproduce, but then again as a result of the culturing conditions, no values between 0 and 1 ( $r$ equals 0 ) will be found, and all values will be larger than one. This would not make the curve-fitting procedure, as carried out in this paper, any easier. Total reproductive output, $R_{0}$, does not take into account the time needed to reproduce, and hence is not suitable as a measurement of fitness either. Fitness is a relative quantity, describing the relative contribution of a certain individual or clone to the next generation, and in most studies values of $r$ are used to compare the reaction of identical individuals to different experimental conditions (e.g. Weider, 1993; Spaak \& Hoekstra, 1995), or to compare different animals under similar conditions (e.g. Ebert \& Jacobs, 1991; Spitze, 1992). Although it should be kept in mind that $r$ does not scale linearly with $\lambda$, and hence that taking $r$ as a fitness measure could bias the results, the relative differences between both estimates will be small, because $r$ is usually close to zero. As argued in the Materials and Methods section, an optimal weight of offspring yielding a value of $r$ smaller then zero would result in a population going to extinction. A computed optimum offspring size resulting in a negative $r$ is therefore outside the domain, and cannot occur. The optimum offspring size should thus be in the range where $r>0$.

Tessier \& Consolatti (1991) reported differences in quality between offspring produced at different food levels: they observed that neonates born at the lowest food level $\left(\approx 0.05 \mathrm{mg} \mathrm{Cl}^{-1}\right)$ had a higher relative nitrogen content than neonates produced at higher food levels. In contrast to these findings, I did not observe quality differences between offspring produced at different food concentrations. Although food quality has been reported to affect egg quality (Müller-Navarra, 1993), my results indicate that this is not the case for differences in food quantity, at least for the rather crude measures of offspring quality used here. Further research should clarify whether indeed differences between offspring produced by females grown at different food levels are only quantitative in nature, or whether more sophisticated measures (e.g. fatty acid spectrum) would reveal also qualitative differences.

The explained variance of the curves fitted through the data relating offspring weight with offspring fitness was low, but significant (Table 2). Generally, it has 


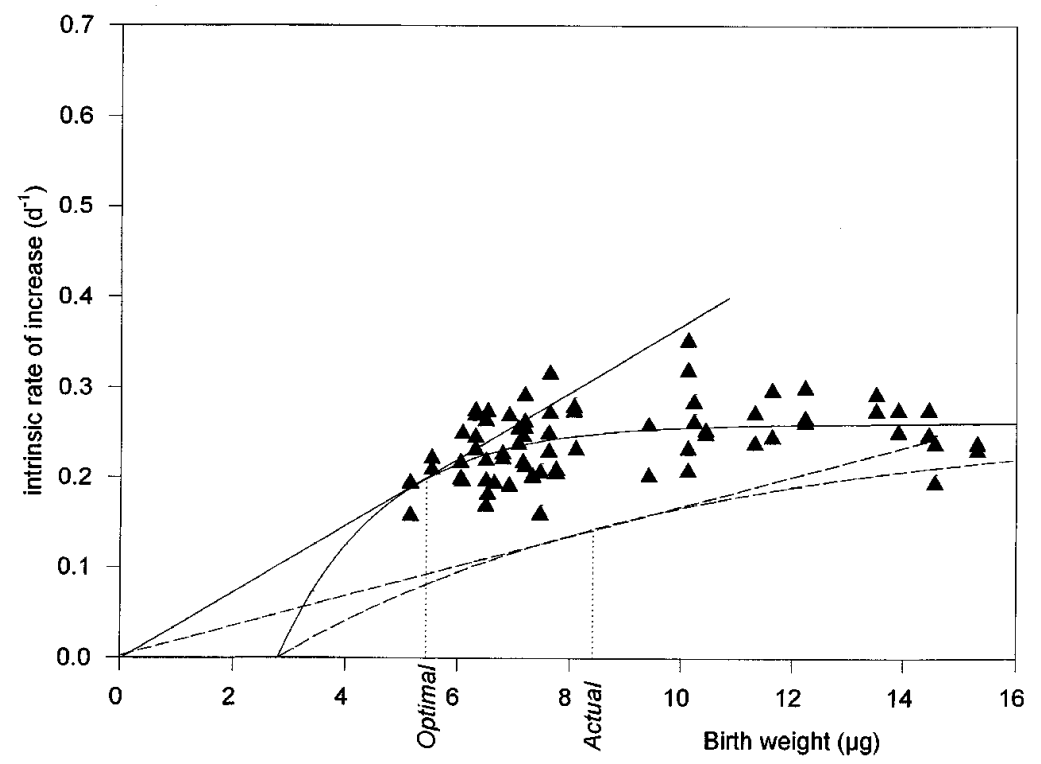

Figure 4. An example of a refit of the function: $f=f_{\mathrm{m}}\left\{1-\mathrm{e}^{-k\left(n-n_{\mathrm{o}}\right)}\right\}$, for animals grown at $0.4 \mathrm{mg} \mathrm{C} 1^{-1}$, with the parameters $k$ and $n_{0}$ changed in such a way that the observed average offspring weight becomes the optimal offspring weight (dotted line).

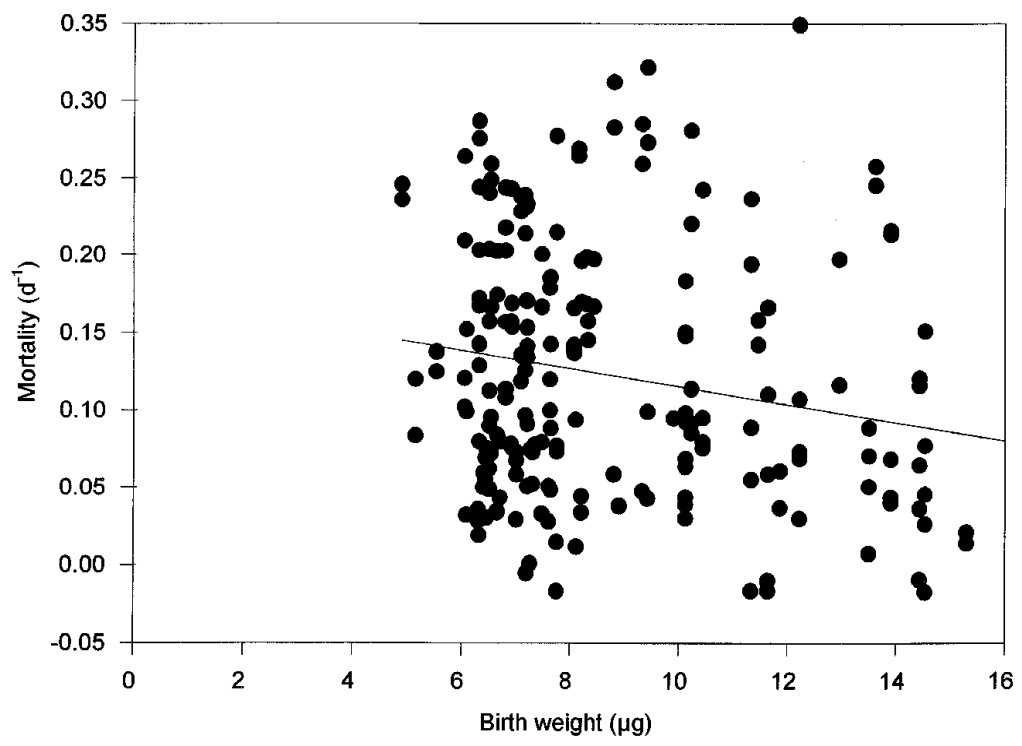

Figure 5. Mortality $\left(\mathrm{d}^{-1}\right)$ needed to explain the discrepancy between optimal offspring weight and observed offspring weight.

been found that the smallest offspring are produced at the highest food levels, while larger offspring are produced at lower food levels (e.g. Tessier \& Consolatti, 1991; Glazier, 1992; Guisande \& Gliwicz, 1992). Hence, it was to be expected that fitting the curves for animals cultured at the highest and at the lowest food levels would prove to be most difficult: at the high- est food level even the smallest neonates were not 'too small' for this food level, while at the lowest food level there were no individual newborn that were 'too large'. Even at the two intermediate food levels variation was large, and also at these food levels animals that were too small for these food concentrations seemed to be lacking. It could be argued that, given the large varia- 
tion in the observed $r$-values, fitting a three parameter model through the data is not valid, and that instead the more parsimonious solution, i.e. linear regression, should have been chosen. This was not done, as fitness of the juveniles cannot increase indefinitely, but rather should show some maximum value. The main problem, however, was the large variation in the data, and the observation that very few offspring seemed to be too small for their environment. The causes of this are unclear. On the one hand, it could have been the case that the mothers used in these experiments were badly fed, leading to the production of large offspring. However, Lampert (1993) reported that eggs with a diameter of $0.22 \mathrm{~mm}$ always failed to hatch. Using the relationships between volume and dry weight established in the first experiment, this would translate in a dry weight of around $4 \mu \mathrm{g}$. Figure 2 shows that a number of the offspring produced in this study were close to this value, so most likely it was not the case that all offspring used in this experiment were too large, but rather it seems to be that in this study differences in initial weight of the offspring are rather unimportant, and that only the actual food level plays a role in the determination of $r$, an observation contradicted by a substantial body of literature, showing maternal effects in daphnids (e.g. Lynch \& Ennis, 1983; Lampert, 1993).

The values computed for $n_{0}$ were smaller than the value for the minimum viable offspring reported by Lampert (1993) of $4 \mu \mathrm{g}$. Bell (1983) also reported for Daphnia pulex that eggs smaller than a certain volume never hatched. This would imply that my estimates for $n_{r m o}$ are too high, which would imply that the computed values for optimal offspring weight are underestimated. Alternatively, the value of minimum viable egg weight as estimated by Lampert (1993) might be too small as these experiments were done in vitro, with a total hatching success of only $50 \%$. This value is certainly lower than in vivo, and it could be the case that the smaller eggs would have hatched in the broodpouch.

As only a few studies have focused on size-selective mortality in the field (Vijverberg \& Richter, 1982; Boersma et al., 1996) it is difficult to judge whether my estimates of the differences in mortality needed to make the actual average birth weight the optimal birth weight are realistic. This would involve a very detailed study of the fate of newborn daphnids with size differences which are rather small. Using the paper of Vijverberg et al. (1993), I estimated how large the differences in mortality would be between the 15 smallest individu- als produced in this study and the 15 largest individuals (ranked according to their lengths). In summer, these differences are in the order of $0.1 \mathrm{~d}^{-1}$, a value comparable to the difference in mortality I computed, and hence it is conceivable that these differences in natural mortality could explain the discrepancy between the observed average weight and the computed optimal weight. This difference in mortality would then comprise of either selective predation on smaller individuals (e.g. by invertebrate predators) or higher nonpredation mortality rates of smaller individuals.

It seems to be the case that especially at the higher food levels, neonates are heavier than the computed optimal offspring weights. The question of size of the offspring produced by daphnids cultured on different food levels has been addressed by a number of workers (e.g. Tessier \& Consolatti, 1989, 1991; Glazier, 1992; Guisande \& Gliwicz, 1992; Ebert, 1993, 1994; Boersma, 1995, 1997), but thus far only a few studies have investigated the relationship between initial offspring size and fitness (e.g. Lynch \& Ennis, 1983). Only Tessier \& Consolatti (1991) tried to link these traits in a more formal way. In an earlier study (Boersma, 1997), I analysed the effect of maternal age on offspring weight and fitness, and observed that, although primiparous females produce the smallest offspring, these neonates had the highest fitness per unit effort. Therefore I concluded that the youngest females produced the optimal offspring weight. The results found in the current study, however, would implicate that even these small offspring produced by the first adult instar females are larger than the optimal weight, and that producing even smaller offspring would yield a higher parental fitness. It is difficult to envisage that daphnids, which frequently experience periods of high food abundance, are suboptimally adapted to their environment, as is suggested by my finding that especially at higher food levels they produce offspring larger than the computed optimal offspring weight. The mean observed offspring weight of the animals cultured at carbon concentrations of $0.2 \mathrm{mg} \mathrm{l}^{-1}$ was similar to the computed optimal weight, whereas at the highest food level the difference amounted to a factor two. This might be a result of an incomplete trade-off between size and number of offspring at these food levels. Indeed, Ebert (1993) reported that the negative correlation between size and number of offspring is strongest at lower food levels, and close to zero when food conditions are good. Although Ebert \& Yampolsky (1993) suggested that oocytes are originally produced in excess, so that the actual egg number might be adjusted downward, it 
is unknown as yet how many oocytes are actually produced. It is conceivable that especially at higher food levels, the number of oocytes, rather than the amount of energy available is limiting the number of eggs produced. This means that the animals have excess energy, which might be distributed to the available oocytes. This 'luxus-nourishment' of the eggs would then result in offspring with a size larger than the optimal size.

Environmental conditions for daphnids in the field are rather unstable. High levels of edible algae at one moment in time can be followed by much lower levels only a few days later, caused by the rapid depletion of food as a result of high densities of zooplankton (e.g. Jeppesen et al., 1990). Moreover, it has become increasing clear that aquatic environments are not as homogenous as previously assumed, but that many zooplankton and phytoplankton populations show a high degree of patchiness in their distributions (George \& Edwards, 1976; Harris, 1980), with, as was reported by Malone \& McQueen (1983) differences of upto a factor three in chlorophyll content within a distance of a few meters. This implies that a slight horizontal or vertical movement of the daphnids could lead to different feeding conditions rather rapidly. As a result, it may not always be the best strategy to adjust completely to concurrent food conditions, as these may change within a few days. Egg weight and number are determined around 0.6 developmental units (instar durations) before they are actually deposited into the broodchamber (e.g. Bradley et al., 1991; Ebert \& Yampolsky, 1993; Stibor, 1995). This means that the timelag between the determination of the weight of the offspring and their release from the broodchamber is 1.6 instar durations, typically around 5.5 days at $20{ }^{\circ} \mathrm{C}$. Larger offspring have higher starvation resistance (Threlkeld, 1976; Tessier et al., 1983), and as a result of the shape of the curves relating parental fitness with effort per offspring (see also Smith \& Fretwell, 1974), the parental fitness loss of producing offspring which are slightly heavier than would be optimal is much lower than the fitness loss associated with the production of offspring which are slightly lighter than the optimal weight. As the likelihood of deteriorating food conditions is obviously greater at high than at low food levels, the advantage of producing larger-thanoptimal-sized offspring is also larger under high food conditions. At low food levels, energy is limiting and producing overweight offspring might imply producing one offspring less, which would obviously decrease parental fitness substantially. In addition, it was suggested that offspring size at low food levels might be limited by other factors, such as the existence of some maximal offspring size (e.g. Ebert, 1994; Boersma, 1995), which would make it impossible to invest extra energy in individual offspring for animals grown under low food conditions.

In conclusion, in this study I observed that especially at higher food levels the difference between observed offspring weights and the calculated optimal offspring weight is large. The most likely explanation for this observation is that daphnids experiencing high food conditions produce these slightly obese offspring to avoid possible starvation in the case of degenerating food conditions. The experiments in this paper were carried out with one laboratory clone of Daphnia magna. Obviously, clones with different backgrounds may react differently, and hence the results obtained here should be corroborated by experiments with a range of clones coming from different locations. It would be of interest to investigate whether clones from more instable habitats produce offspring that are overweight to a higher degree than clones from more stable habitats.

\section{Acknowledgements}

I thank Herwig Stibor for his help with the analysis of the fat content of the Daphnia eggs and Hinnerk Boriss, Dieter Ebert, Winfried Lampert, Klaus Plath, Larry Weider and two anonymous referees for their comments on the manuscript.

\section{References}

Bell, G., 1983. Measuring the cost of reproduction. 3. The correlation structure of the early life history of Daphnia pulex. Oecologia 60: 378-383.

Boersma, M., 1995. The allocation of resources to reproduction in Daphnia galeata: Against the odds? Ecology 76: 1251-1261.

Boersma, M., 1997. Offspring size and parental fitness in Daphnia magna. Evol. Ecol.: 11: 439-450.

Boersma, M., O. F. R. van Tongeren \& W. M. Mooij, 1996. Seasonal patterns in the mortality of Daphnia species in a shallow lake. Can. J. Fish. aquat. Sci. 53: 18-28.

Bradley, M. C., D. J. Baird \& P. Calow, 1991. Mechanisms of energy allocation to reproduction in the cladoceran Daphnia magna Straus. Biol. J. Linn. Soc. 44: 325-333.

De Meester, L., 1994. Life histories and habitat selection in Daphnia - divergent life histories of D. magna clones differing in phototactic behaviour. Oecologia 97: 333-341.

Ebert, D., 1993. The trade-off between offspring size and number in Daphnia magna - the influence of genetic, environmental and maternal effects. Arch. Hydrobiol. Suppl. 90: 453-473. 
Ebert, D., 1994. Fractional resource allocation into few eggs - Daphnia as an example. Ecology 75: 568-571.

Ebert, D. \& J. Jacobs, 1991. Differences in life-history and aging in 2 clonal groups of Daphnia cucullata Sars (Crustacea, Cladocera). Hydrobiologia 225: 245-253.

Ebert, D. \& L. Y. Yampolsky, 1992. Family planning in Daphnia: when is clutch size determined? Russian J. aquat. Ecol. 2: 143148.

George, D. G. \& R. W. Edwards, 1976. The effect of wind on the distribution of chlorophyll a and crustacean plankton in a shallow eutrophic reservoir. J. appl. Ecol. 13: 667-692.

Glazier, D. S., 1992. Effects of food, genotype, and maternal size and age on offspring investment in Daphnia magna. Ecology 73: 910-926.

Guisande, C. \& Z. M. Gliwicz, 1992. Egg size and clutch size in 2 Daphnia species grown at different food levels. J. Plankton Res. 14: $997-1007$.

Harris, G. P., 1980. Temporal and spatial scales in phytoplankton ecology. Mechanisms, methods, models, and management. Can. J. Fish. aquat. Sci. 37: 877-900.

Jeppesen, E., M. Søndergaard, O. Sortkjær, E. Mortensen \& P. Kristensen, 1990. Interactions between phytoplankton, zooplankton and fish in a shallow, hypertrophic lake: a study of phytoplankton collapses in Lake Søbygård, Denmark. Hydrobiologia 191: $149-164$.

Lack, D., 1947. The significance of clutch size. Ibis 89: 309-352.

Lampert, W., 1993. Phenotypic plasticity of the size at first reproduction in Daphnia: the importance of maternal size. Ecology 74: 1455-1466.

Lampert, W. \& I. Trubetskova, 1996. Juvenile growth rate as a measure of fitness in Daphnia. Funct. Ecol. 10: 631-635.

Lampert, W., R. D. Schmitt \& P. Muck, 1988. Vertical migration of freshwater zooplankton: test of some hypotheses predicting a metabolic advantage. Bull. Mar. Sci. 43: 620-640.

Lynch, M. \& R. Ennis, 1983. Resource availability, maternal effects, and longevity. Exper. Geront. 18: 147-165.

Malone, B. J. \& D. J. McQueen, 1983. Horizontal patchiness in zooplankton populations in two Ontario kettle lakes. Hydrobiologia 99: 101-124.

Müller-Navarra, D., 1993. Quantifizierung von Nahrungsqualität für herbivores Zooplankton. PhD Thesis, University of Kiel, $137 \mathrm{pp}$.

Parker, G. A. \& M. Begon, 1986. Optimal egg size and clutch size: effects of environment and maternal phenotype. Am. Nat. 128: 573-592.
Ridley, M., 1993. Evolution. Blackwell. Boston, 670 pp.

Smith C. C. \& S. D. Fretwell, 1974. The optimal balance between size and number of offspring. Am. Nat. 108: 499-506.

Sokal, R. R. \& F. J. Rohlf, 1981. Biometry. Freeman and Company, San Francisco.

Spaak, P. \& J. R. Hoekstra, 1995. Life history variation and the coexistence of a Daphnia hybrid with its parental species. Ecology 76: 553-564.

Spitze, K., 1992. Predator-mediated plasticity of prey life history and morphology - Chaoborus americanus predation on Daphnia pulex. Am. Nat. 139: 229-247.

Stearns, S. C., 1992. The Evolution of Life Histories. Oxford University Press, Oxford, 249 pp.

Stibor, H., 1995. Chemische Informationen in limnischen RäuberBeute Systemen: Der Effekt von Räubersignalen auf den Lebenszyklus von Daphnia spp. (Crustacea: Cladocera). PhD Thesis, University of Kiel, $150 \mathrm{pp}$.

Tessier, A. J. \& N. L. Consolatti, 1989. Variation in offspring size in Daphnia and consequences for individual fitness. Oikos 56: 269-276.

Tessier, A. J. \& N. L. Consolatti, 1991. Resource quantity and offspring quality in Daphnia. Ecology 72: 468-478.

Tessier, A. J., L. L. Henry, C. E. Goulden \& M. W. Durand, 1983. Starvation in Daphnia: Energy reserves and reproductive allocation. Limnol. Oceanogr. 28: 667-676.

Threlkeld, S. T., 1976. Starvation and the size structure of zooplankton communities. Freshwat. Biology 6: 489-496.

van Noordwijk, A. J. \& G. de Jong, 1986. Acquisition and allocation of resources: their influence on variation in life history tactics. Am. Nat. 128: 137-142.

Vijverberg, J. \& A. F. Richter, 1982. Population dynamics and production of Daphnia hyalina Leydig and Daphnia cucullata Sars in Tjeukemeer. Hydrobiologia 95: 235-259.

Vijverberg, J., R. D. Gulati \& W. M. Mooij, 1993. Food-web studies in shallow eutrophic lakes by the Netherlands Institute of Ecology: Main results, knowledge gaps and new perspectives. Neth. J. aquat. Ecol. 27: 35-49.

Weider, L. J., 1993. Niche breadth and life history variation in a hybrid Daphnia complex. Ecology 74: 935-943.

Winkler, D. W. \& K. Wallin, 1987. Offspring size and number: a life history model linking effort per offspring and total effort. Am. Nat. 129: 708-720. 